\title{
Expansión de la invasión del Visón Norteamericano (Neovison vison) en la Reserva de la Biosfera de Cabo de Hornos, Chile
}

\author{
Invasive expansion of the American Mink (Neovison vison) \\ in the Cape Horn Biosphere Reserve, Chile
}

Ramiro D. Crego ${ }^{1,3,5}$, Jaime E. Jiménez $z^{1,2,3,4,5}$

\& Ricardo Rozzi ${ }^{1,2,3,4,5}$

La Reserva de la Biosfera Cabo de Hornos $(\mathrm{RBCH})$ se ubica en el extremo sur del continente sudamericano. Dado el gran remanente de vegetación nativa, junto a la baja fragmentación y baja densidad-poblacional humana, la región se encuentra catalogada como una de las últimas 24 áreas prístinas del planeta (Mittermeier et al. 2003). La RBCH está embebida en la ecoregión de los Bosques Sub-Antárticos caracterizada por la presencia de coigües de Magallanes (Nothofagus betuloides) (Rozzi et al. 2006). Una de las mayores amenazas a la conservación de esta área son las invasiones biológicas, dado que numerosas especies exóticas han logrado establecerse en la región (Anderson et al. 2006; Valenzuela et al. 2014).

Una de las invasiones más recientes y problemáticas en Cabo de Hornos es la del visón norteamericano (Neovison vison), un mustélido de hábitos semi-acuáticos, que es originario de América del Norte (Larivière, 1999). Diversos escapes accidentales $\mathrm{o}$ intencionales desde granjas de producción peletera ocurrieron en varios sitios de la Patagonia argentina y chilena a partir de la década de 1930 (Jaksic et al. 2002; Fasola et al. 2010). En lo que respecta a la población invasora del Cabo de Hornos, varios individuos fueron liberados desde granjas entre 1948 y 1953 del lado argentino de Tierra del Fuego así como también en 1961 en la península Mitre del lado chileno (Lizarralde \& Escobar, 2000). Desde estos sitios el visón expandió su distribución por la isla de Tierra del Fuego, cruzando luego el canal Beagle, siendo detectado por primera vez en la isla Navarino en el año 2001 (Rozzi \& Sherriffs, 2003). Para el año 2003 ya existían registros de presencia de visón en la costa sur de isla Navarino (Rozzi \& Sherriffs, 2003). Subsecuentemente la especie se dispersó por otras islas del archipiélago tales como las islas Hoste en 2005 (Anderson et al. 2006), Lennox en 2009 (Davis et al. 2012) y Gordon y Gabriel en 2012 (Valenzuela et al. 2014). Sin embargo, hasta la fecha la cordillera de Darwin y su casquete de hielo y glaciares parecían ser una barrera eficiente que mantenía una zona importante de Tierra del Fuego y la $\mathrm{RBCH}$ libre de este depredador. El visón norteamericano está catalogado dentro de las 100 especies invasoras más dañinas de Chile (SAG 2011) ${ }^{1}$ y numerosos estudios reportan el impacto negativo sobre la fauna nativa (Schüttler et al. 2009; Maley et al.

1 Department of Biological Sciences, University of North Texas, Denton, TX, USA. ramirocrego84@gmail.com $\bowtie$

2 Department of Philosophy and Religion Studies, University of North Texas, Denton, TX, USA.

3 Instituto de Ecología y Biodiversidad, Departamento de Ciencias Ecológicas, Facultad de Ciencias, Santiago, Chile.

4 Universidad de Magallanes, Punta Arenas, Chile.

5 Programa de Conservación Biocultural Subantártica, Chile.

1 SERVICIO AGRICOLA Y GANADERO, Subdepartamento de vida silvestre DIPROREN 2011. LEGISLACIÓN La Ley de Caza y su Reglamento, 12va edn. Unidad comunicación y prensa, SAG, Santiago, Chile. 


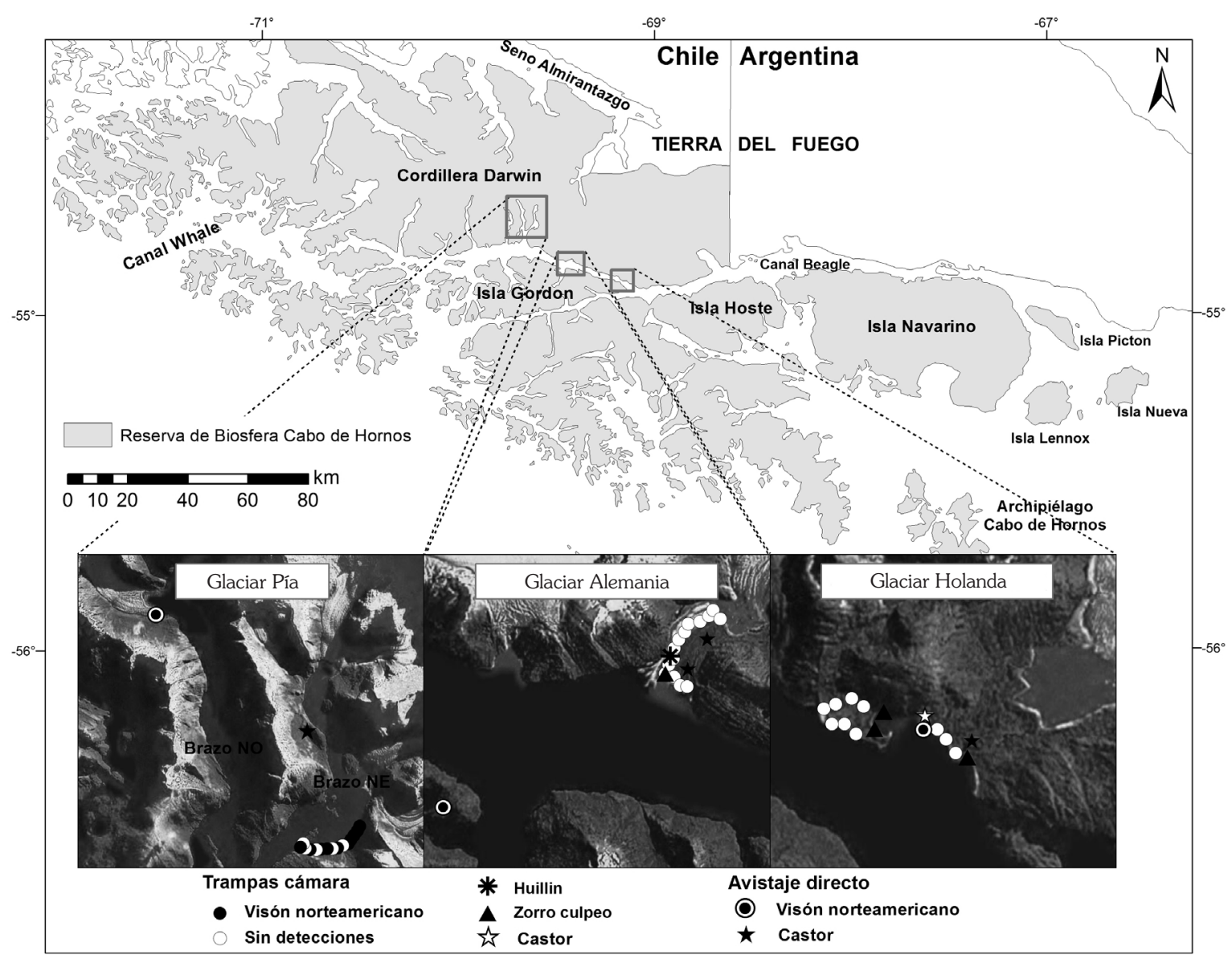

Fig. 1. El mapa muestra la Reserva de la Biosfera Cabo de Hornos, Chile, con los sitios muestreados y lugares donde se detectó la presencia de visones, castores, zorros culpeo y hullines, tanto mediante trampas cámara como por avistajes directos.

2011; Liljesthröm et al. 2013; Valenzuela et al. 2013; Crego et al. 2014; Jiménez et al. 2014). Sin embargo, en la región aun no se han tomado medidas de control sostenidas en el tiempo que permitan un control eficiente de la población. En esta nota reportamos la continua expansión del visón norteamericano a nuevos sitios de la $\mathrm{RBCH}$, como un llamado a la pronta acción para preservar la biodiversidad de uno de los últimos sitios prístinos del planeta.

Durante el mes de enero de 2015 realizamos una expedición al brazo norte del canal Beagle en el Parque Nacional D'Agostini con el objetivo de monitorear la presencia del visón en la zona de los glaciares Pía ( $54^{\circ} 47^{\prime}$ S; $69^{\circ} 35^{\prime}$ O), Alemania ( $54^{\circ}$

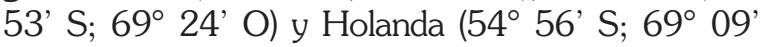
O) (Fig. 1). La idea original fue obtener información de un sitio presunto de estar libre de visones, para comparar su impacto sobre la biodiversidad con estudios en la isla Navarino. Usamos dos metodologías para determinar presencia/ausencia de visón en cada sitio. En primer lugar se colocaron 15 trampas cámara (Bushnell Trophy Cam, Modelo 119446) en zonas costeras y de bosque del glaciar Pía y Alemania, y 14 en la zona del glaciar Holanda (Fig. 1), espaciadas $200 \mathrm{~m}$ entre sí y cebadas con pescado enlatado. Las cámaras estuvieron activas por un período de 3 noches y 2 días. Asimismo, en cada sitio dos personas realizaron recorridos exploratorios no sistemáticos buscando activamente signos tales como huellas, fecas u observaciones de individuos que documentaran la presencia de visones.

En total registramos 45 fotos de 5 especies de mamíferos (considerando una hora de diferencia entre detecciones como eventos independientes; Tabla 1). La presencia de visones mediante este 
Tabla1. Lista de mamíferos detectados en el brazo norte del canal de Beagle en la Reserva de la Biosfera Cabo de Hornos, durante el mes de Enero de 2015.

\begin{tabular}{|c|c|c|c|}
\hline Nombre común & Nombre científico & Sitio & Método de identificación \\
\hline \multirow[t]{3}{*}{ Visón norteamericano } & Neovison vison & Brazo NE glaciar Pía & $\begin{array}{l}\text { Trampa cámara, avistamiento directo, fecas } \\
\text { y huellas. }\end{array}$ \\
\hline & & Brazo NO glaciar Pía & Avistamiento directo. \\
\hline & & Glaciar Holanda & Individuo muerto y huellas. \\
\hline \multirow[t]{3}{*}{ Castor } & Castor canadensis & Brazo NE glaciar Pía & Avistamiento directo, castoreras activas. \\
\hline & & Glaciar Alemania & Avistamiento directo, castoreras activas. \\
\hline & & Glaciar Holanda & $\begin{array}{l}\text { Trampa cámara, avistamiento directo, } \\
\text { castoreras activas. }\end{array}$ \\
\hline Rata almizclera & Ondatra zibethicus & Glaciar Holanda & Fecas y huellas. \\
\hline \multirow[t]{2}{*}{ Zorro culpeo } & Lycalopex culpaeus & Glaciar Alemania & Trampa cámara, fecas y huellas. \\
\hline & & Glaciar Holanda & Trampa cámara, fecas y huellas. \\
\hline Huillin & Lontra provocax & Glaciar Alemania & Trampa cámara. \\
\hline
\end{tabular}

método se registró sólo en el brazo noreste del glaciar Pía (Fig. 1). Se registraron un total de 35 visitas de visones a las cámaras (Tabla 1). Identificamos con seguridad 4 individuos diferentes en base a diferencias de color y tamaño, pero es probable que fueran más, ya que es muy difícil individualizar a los visones en base a las fotografías. Sin embargo, las fotos documentaron la presencia de individuos juveniles junto a adultos (Fig. 2), los cuales fueron diferenciados en las fotografías por tamaño y comportamiento. Los visones son animales solitarios y solo se los ve en números de 3 o más cuando las madres andan junto a sus crías (Larivière, 1999). Esto indica que se trataría de una población reproductiva. No obtuvimos registros de visones en las trampas cámara en la zona del glaciar Alemania, aunque destacamos la detección de un ejemplar de huillín (Lontra provocax) y de zorro culpeo (Lycalopex culpaeus). Por último, en la zona del glaciar Holanda solo registramos la actividad de castores (Castor canadensis) y zorro culpeo.

En cuanto a los recorridos a pie, avistamos 2 ejemplares de visón en el brazo noreste del glaciar Pía y un ejemplar en el brazo noroeste del glaciar Pía, este último caminando junto al glaciar. Además, encontramos un animal muerto junto a la costa en la zona del glaciar Holanda y avistamos un individuo caminando por el suelo del bosque en la costa de Caleta Morning, en isla Gordon. En resumen, registramos presencia
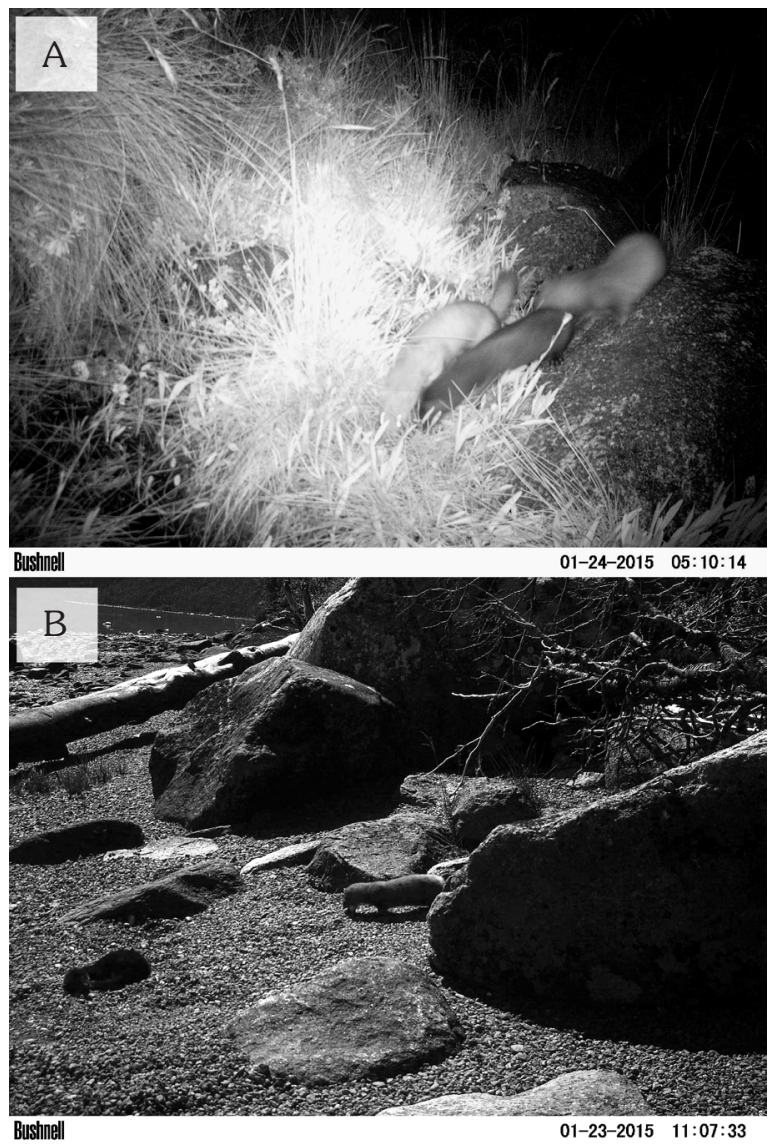

Fig. 2. Fotos de visón norteamericano en glaciar Pía, Reserva de la Biosfera Cabo de Hornos, Chile. La foto A muestra tres individuos, un adulto con dos juveniles, mientras que la foto $\mathrm{B}$ muestra dos juveniles en la playa. 
de visón en el glaciar Pía y Holanda e isla Gordon, no teniendo registro alguno para el glaciar Alemania (Fig. 1). Además registramos la presencia de castoreras activas en los tres sitios muestreados (Fig. 1) y presencia de huellas y fecas de ratas almizcleras (Ondatra zibethicus) en las castoreras del glaciar Holanda (Tabla 1). Por último avistamos un individuo de visón en la costa norte de isla Hoste.

En esta nota reportamos los primeros registros de visón norteamericano en la zona suroeste de Tierra del Fuego. La población estaría reproductiva y es probable que siga expandiéndose por el resto de la $\mathrm{RBCH}$. Estimamos que los visones estarían dispersándose por la costa del canal Beagle, pero también podrían cruzar el mismo desde isla Gordon. Tampoco se descarta la hipótesis que estén siendo dispersados accidentalmente a bordo de barcos de pescadores (Valenzuela et al. 2014). Actualmente, son pocas las islas de la $\mathrm{RBCH}$ que estarían libres de visones: islas del archipiélago de Cabo de Hornos y las islas al sur del canal Whale, aunque esto podría deberse a la falta de monitoreos por lo que futuros muestreos serán importantes para confirmar su presencia o ausencia.

Los efectos del visón sobre la fauna nativa son claros y significativos. Los visones representan una gran amenaza para los roedores nativos y las aves del bosque (Crego et al. 2014), incluso para el carpintero magallánico (Campephilus magellanicus) (Jiménez et al. 2014) que habitan la $\mathrm{RBCH}$. Durante la expedición se detectó una alta abundancia de churrines (Scytalopus magellanicus) (Jiménez et al. enviado). Esta ave solía ser abundante en isla Navarino hasta comienzos de la década del 2000, y hoy es raramente vista o escuchada, atribuyéndose su declinación a su depredación por visones (Rozzi \& Jiménez, 2014).

Consideramos de extrema urgencia $e$ importancia que se tomen medidas para controlar y monitorear al visón que ya ha invadido la zona núcleo de la $\mathrm{RBCH}$. Los programas de control del visón en la región están focalizados principalmente sobre la línea de costa de la isla Navarino y en menor medida sobre los cursos de agua de los principales ríos (Caicheo, 2010). Sin embargo, estos no son efectivos ya que son de bajo impacto y esporádicos, quizás debido a los problemas logísticos, sociales y burocráticos que atentan y dificultan la continua implementación de tales medidas. Si bien trabajos en otras latitudes del mundo muestran que el control o incluso erradicación de la especie es posible (Bonesi \& Palazon, 2007; Melero et al. 2010; Bryce et al. 2011), para que esto ocurra en la $\mathrm{RBCH}$ se necesitan tomar medidas de control efectivas con extrema urgencia. Estas medidas permitirán proteger la biodiversidad nativa cuando aún sea posible. y así evitar la extinción local de poblaciones de aves y micromamíferos y asegurar la conservación de la $\mathrm{RBCH}$, una de las ultimas regiones prístinas del planeta.

\section{AGRADECIMIENTOS}

Este estudio fue realizado con permiso de CONAF (Resolución $N^{\circ}$ : 711/2014) y fue financiado con subsidios del Programa de Conservación Biocultural Subantártica de la Universidad de North Texas, Universidad de Magallanes, y del Instituto de Ecología y Biodiversidad de Chile (ICM P05-002 y CONICYT PFB-23), además del Toulouse Graduate School Program de la Universidad de North Texas. Agradecemos el apoyo de la Fundación Omora y la Dra. Francisca Massardo. Deseamos también expresar nuestros agradecimientos a Omar Barroso y Brian Milstead por su colaboración en los trabajos de campo y a dos revisores anónimos por sus valiosos aportes que mejoraron el manuscrito.

\section{LITERATURA CITADA}

Anderson, C. B., Rozzi, R., Torres-Mura, J. C., McGehee, S. M., Sherriffs, M. F., Schüttler, E. \& Rosemond, A. D. (2006). Exotic vertebrate fauna in the remote and pristine Sub-Antarctic Cape Horn Archipelago, Chile. Biodiversity and Conservation, 15, 3295-3313.

Bonesi, L \& Palazon, S. (2007). The American mink in Europe: Status, impacts, and control. Biological Conservation, 134, 470-483.

Bryce, R., Oliver, M. K., Davies, L., Gray, H., Urquhart, J. \& Lambin, X. (2011). Turning back the tide of American mink invasion 
at an unprecedented scale through community participation and adaptive management. Biological Conservation, 144, 575-583.

Caicheo, A. M. (2010). Plan de acción para el control del visón norteamericao (Neovison vison), especie invasora en la isla Navarino, Reserva de Biosfera Cabo de Hornos, XIla Región, Chile. Preparado para el Servicio Agrícola y Ganadero, Magallanes, Antártica Chilena. Puerto Williams, Chile.

Crego, R. D., Jiménez, J. E., Soto, C., Barroso, O. \& Rozzi, R. (2014). Tendencias poblacionales del visón norteamericano invasor (Neovison vison) y sus principales presas nativas desde su arribo a isla Navarino, Chile. Boletín de la Red Latinoamericana para el Estudio de Especies Invasoras, 4, 4-18.

Davis, E. F., Anderson, C. B., Valenzuela, A. E. J., Cabello, J. L. \& Soto, N. (2012). American mink (Neovison vison) trapping in the Cape Horn Biosphere Reserve: enhancing current trap systems to control an invasive predator. Acta Zoologica Fennica, 49, 18-22.

Fasola, L., Muzio, J. \& Chehébar, C. (2010). Range expansion and prey use of American mink in Argentinean Patagonia: dilemmas for conservation. European Journal of Wildlife Research, 57, 283-294.

Jaksic, F. M., Iriarte, J. A., Jiménez, J. E. \& Martínez, D. R. (2002). Invaders without frontiers: cross-border invasions of exotic mammals. Biological Invasions, 4, 157173.

Jiménez, J. E., Crego, R. D., Soto, G. E., Román, I., Rozzi, R. \& Vergara, P. M. (2014). Potential impact of the alien American mink (Neovison vison) on Magellanic woodpeckers (Campephilus magellanicus) in Navarino Island, Southern Chile. Biological Invasions, 16, 961-966.

Larivière, S. (1999). Mustela vison. Mammalian Species, 608, 1-9.

Liljesthröm, M., Schiavini, A., Sáenz Samaniego, R., Fasola, L. \& Raya Rey, A. (2013). Kelp Geese (Chloephaga hybrida) and Flightless Steamer-Ducks (Tachyeres pteneres) in the Beagle Channel: the importance of islands in providing nesting habitat. The Wilson Journal of Ornithology, 125, 583-591.

Lizarralde, M. S. \& Escobar, J. M. (2000). Mamíferos exóticos en la Tierra del Fuego. Ciencia Hoy, 10,52-63.

Maley, B. M., Anderson, C. B., Stodola, K. \& Rosemond, A. D. (2011). Identyfing native and exotic predators of ground-nesting songbirds in Subantarctic forests in Southern Chile. Anales del Instituto de la Patagonia, 39,51-57.

Melero, Y., Palazón, S., Bonesi, L. \& Gosálbez, J. (2010). Relative abundance of culled and not culled American mink populations in northeast Spain and their potential distribution: are culling campaigns effective? Biological Invasions, 12, 3877-3885.

Mittermeier, R. A., Mittermeier, C. G., Brooks, T. M., Pilgrim, J. D., Konstant, W. R., da Fonseca, G. A. B. \& Kormos, C. (2003). Wilderness and biodiversity conservation. PNAS, 100, 10309-10313.

Rozzi, R. \& Jiménez, J. E. (2014). Magellanic Sub-Antarctic ornithology: First Decade of Bird Studies at the Omora Ethnobotanical Park, Cape Horn Biosphere Reserve, Chile. University of North Texas press, Denton, TX, USA.

Rozzi, R., Massardo, F., Anderson, C. B., Heidinger, K. \& Silander, J. A. (2006). Ten principles for biocultural conservation at the southern tip of the Americas: the approach of the Omora Ethnobotanical Park. Ecology and Society, 11, 43-70.

Rozzi, R. \& Sherriffs, M. F. (2003). El visón (Mustela vison Schreber, Carnivora: Mustelidae), un nuevo mamífero exótico para la Isla Navarino. Anales del Instituto de la Patagonia, 31, 97-104.

Schüttler, E., Klenke, R., McGehee, S., Rozzi, R. \& Jax, K. (2009). Vulnerability of groundnesting waterbirds to predation by invasive American mink in the Cape Horn Biosphere Reserve, Chile. Biological Conservation, 142, 1450-1460.

Valenzuela, A. E. J., Anderson, C. B., Fasola, L. \& Cabello, J. L. (2014). Linking invasive 
exotic vertebrates and their ecosystem impacts in Tierra del Fuego to test theory and determine action. Acta Oecologica, 54,110-118.

Valenzuela, A. E. J., Raya Rey, A., Fasola, L., Sáenz Samaniego, R. A. \& Schiavini, A.
(2013). Trophic ecology of a top predator colonizing the southern extreme of South America: Feeding habits of invasive American mink (Neovison vison) in Tierra del Fuego. Mammalian Biology, 78, 104-110. 